\title{
A Reconfigurable Neural Spike Recording Channel with Feature Extraction Capabilities
}

\author{
Alberto Rodríguez-Pérez, Jesús Ruiz-Amaya, Óscar Guerra and Manuel Delgado-Restituto \\ Institute of Microelectronics of Seville (IMSE-CNM-CSIC) and University of Seville \\ Edificio IMSE-CNM, Avda. Américo Vespucio s/n, 41092-Sevilla, SPAIN. \\ e-mail: alberto@imse-cnm.csic.es Tlf.: +34 954466667 Fax: +34 954466600
}

\begin{abstract}
This paper describes the architecture of a neural spike recording channel with feature extraction capabilities and presents the design of one of its key elements, a reconfigurable 8bit ADC. The ADC can be programmed for different conversion rates and embeds a 0-18dB programmable gain amplifier with discrete gain steps of $3 \mathrm{~dB}$. Simulation results from extracted layout of the ADC, designed in a 130nm CMOS technology, obtain almost 8-bit ENOB at $22.2 \mathrm{kS} / \mathrm{s}$ and $90 \mathrm{kS} / \mathrm{s}$, with a power consumption of $500 \mathrm{nW}$ and $1.8 \mu \mathrm{W}$, respectively.
\end{abstract}

\section{I.INTRODUCTION}

During the last years, there has been a growing interest on the design of neural recording interfaces with wireless transmission capabilities for the untethered measurement of brain activity [1-4]. These interfaces are expected to play a significant role both in clinical (as part of therapeutic procedures in patients with neurological diseases) and neuroscience applications. In order to reduce the data flow through the communication link, there have been proposed neural interfaces which internally provide data reduction mechanisms so that only the clinically relevant information is wirelessly transmitted. These on-chip feature extraction solutions have been implemented both in analogue [1-2] and digital [3-4] domain, but they tend to be bulky and power demanding. This later aspect is particularly troublesome as neural recording interfaces are often remotely powered using inductive transponder links.

In this paper we present a low power neural spike recording channel suitable for raw brain activity acquisition, on the one hand, and detection and characterization of spikes, on the other. The proposed solution employs mixed-signal techniques, and uses a foreground digital calibration process to counteract technology process variations.

One of the key blocks in the proposed architecture is an 8bit reconfigurable Analog-to-Digital Converter (ADC), able to run under different channel operation modes and sampling rates. As an additional feature, the ADC can be also programmed to amplify the incoming signal from 0 to $18 \mathrm{~dB}$. Simulation results from extracted layout show that the ADC only consumes $500 \mathrm{nW}$ (alt., $1.8 \mu \mathrm{W}$ ) when operated at $22.5 \mathrm{kS} / \mathrm{s}$ (alt., $90 \mathrm{kS} / \mathrm{s}$ ). In other respects, the complete neural recording channel operated in signal tracking and spike processing modes consumes 2.1 and $3.3 \mu \mathrm{W}$, respectively (worst-case full transistor-level simulations under PVT variations).

The paper is structured as follows. Section II describes the architecture of the channel and its operation modes, while Section III describes the reconfigurable ADC, which has been

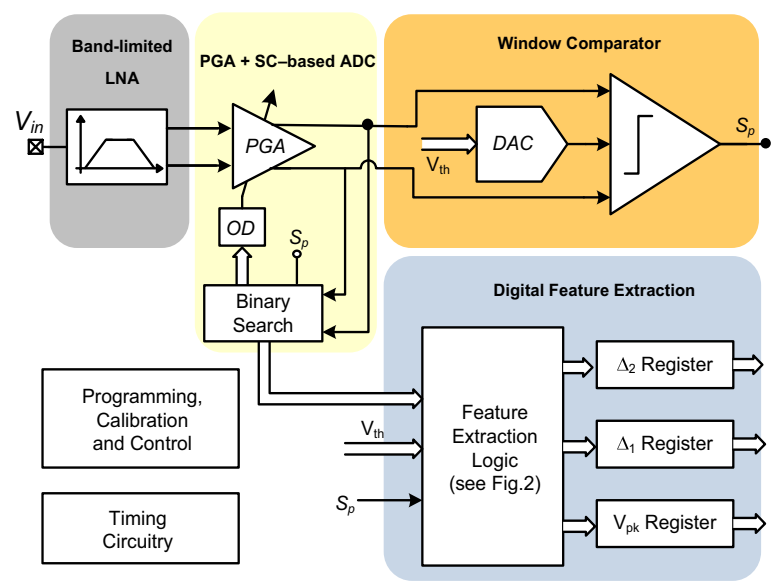

Fig. 1: Simplified neural recording channel architecture.

designed in a 130nm CMOS technology. Section IV gives simulations results of the extracted layout of the ADC and, finally, Section $\mathrm{V}$ concludes the paper.

\section{II.CHANNEL ARCHITECTURE AND OPERATION MODES}

Fig. 1 shows the block diagram of the proposed neural spike recording channel. It consists of a band-limited lownoise amplifier for neural signal acquisition, a reconfigurable Analogue-to-Digital Converter (ADC), a Switched-Capacitor (SC) window comparator and some digital circuitry for control and feature extraction purposes. The ADC is formed by a programmable gain amplifier (PGA) and a binary search algorithm block which emulates the functionality of capacitive DACs in conventional SAR converters.

Three different operation modes can be programmed in the channel. In the calibration mode, the transfer characteristic of the LNA, the gain of the PGA and the threshold value used for spike detection are sequentially adjusted and stored in a local setting memory. In the signal tracking mode, the neural signal is acquired by the LNA and digitized by the ADC. Finally, in the feature extraction mode, neural spikes are on-the-fly detected and characterized by means of Piece-Wise Linear (PWL) functions.

Depending on the channel operation mode, blocks are total or partially powered off for power saving. Moreover, there is not a single clock for the whole system. Instead, active blocks are operated by dedicated clock signals (derived from a master $1.6 \mathrm{MHz}$ reference) with frequencies that depend on the particular system configuration. These and other aspects are briefly described below for each operation mode. 


\section{A. Calibration mode}

In this mode, only the LNA, the ADC and some calibration specific circuitry (not shown in Fig.1) are active. The ADC operates at a $200 \mathrm{kHz}$ clock frequency to provide a throughput rate of $22.5 \mathrm{kS} / \mathrm{s}$ and 8 -bit resolution.

A first step in calibration mode is the tuning of the bandpass characteristic of the LNA to counteract technology process variations. Two programming bits are used to tune the highpass frequency corner and three control bits are used to set the lowpass frequency. The target of the tuning mechanism is to define a signal acquisition passband from about $200 \mathrm{~Hz}$ to $7 \mathrm{kHz}$, suitable for spike recording applications. This is accomplished by isolating the LNA from the microelectrode interface and using the master clock frequency as a reference tuning signal.

Afterwards, the system enters in a long signal tracking phase to consecutively calibrate the gain of the PGA and the threshold voltage for spike detection. In both cases, calibration is realized in a digital manner taking advantage of the ADC available in the system. The aim of the PGA gain adjustment is to scale the amplitude of the LNA output signal to roughly fit the dynamic range of the ADC. To this end, the PGA provides 3 programmable bits to set the gain from 0 to $18 \mathrm{~dB}$ at discrete steps of $3 \mathrm{~dB}$ (this gives a total gain for the channel front-end of $48-66 \mathrm{~dB}$, including the LNA contribution). At the beginning of the calibration, the PGA gain is set to its maximum value while the ADC converts the neural waveform. An auxiliary Overflow Detector (OD) block detects any saturation in the digitized word. If this occurs, the PGA gain is step down to the next lower gain level. The calibration concludes when saturation events are no longer observed.

Finally, the calibration phase concludes by setting the threshold values for spike detection. Given its low computational complexity, essential to reduce the power budget of the channel circuit, the absolute value method has been considered in this work [5]. Similar to the PGA gain adjustment method, the threshold value is also digitally calculated and the result (an 8-bit word) stored in a local memory.

According to our system-level simulations using samples from real human neural recording database [6], about 100 seconds suffice to complete the calibration mode.

\section{B. Signal tracking mode}

In this mode, the signal captured by the neural electrode is conditioned by the LNA and 8-bits digitized by the ADC at a $22.5 \mathrm{kS} / \mathrm{s}$ throughput rate. The output of the ADC is serially read out at a rate of $1.6 \mathrm{MHz}$ to allow for real-time external reconstruction of the neural data.

\section{Feature extraction mode}

This mode provides a data reduction mechanism to reduce the bandwidth of the information transmitted from the channel. Instead of the complete raw data acquired and digitized by the analog front-end as in the previews mode, only some features extracted from the detected spikes are externally transferred. Two phases can be distinguished in this operation mode which are referred to as spike detection and spike processing. They are both shown in the functional diagram of Fig. 2 .

During spike detection, the output of the PGA is directly processed by the window comparator at a clock frequency of $100 \mathrm{kHz}$, whereas the SAR algorithm block is switched-off for power saving. The SC window comparator detects if the voltage magnitude of the PGA output exceeds the threshold value calculated during calibration. A charge redistribution $\mathrm{DAC}$ is used to convert the digital threshold into analogue form. Once a positive or negative spike is detected, control signal $E n$ turns on (it remains in the low state, otherwise), the window comparator is disabled, and the spike processing phase starts.

The purpose of the processing phase is to create a PWL approximation of the spike, as illustrated in Fig. 3. In the case of monophasic spikes, this requires three variables: two time segments to define the peak position, $\Delta_{1}$, and the overall duration of the spike, $\Delta_{2}$; and a measurement of the peak amplitude, $V_{p}$. In the case of biphasic spikes, two sets of the aforementioned variables (one per phase) are required, together with an additional measurement of the separation between the phases, $\Delta_{3}$. All these variables are codified into 8bit digital vectors to give an overall data load of 24 or 56 bits, depending on the spike shape. In this first-order PWL approximation, potential waveform fluctuations during the spike phases are ignored: only absolute maximum or minimum values are considered in the spike representation.

Spike processing is essentially implemented in digital domain. An 8-bit counter, working at $100 \mathrm{kHz}$ and enabled by the En signal, provides the temporal information required by

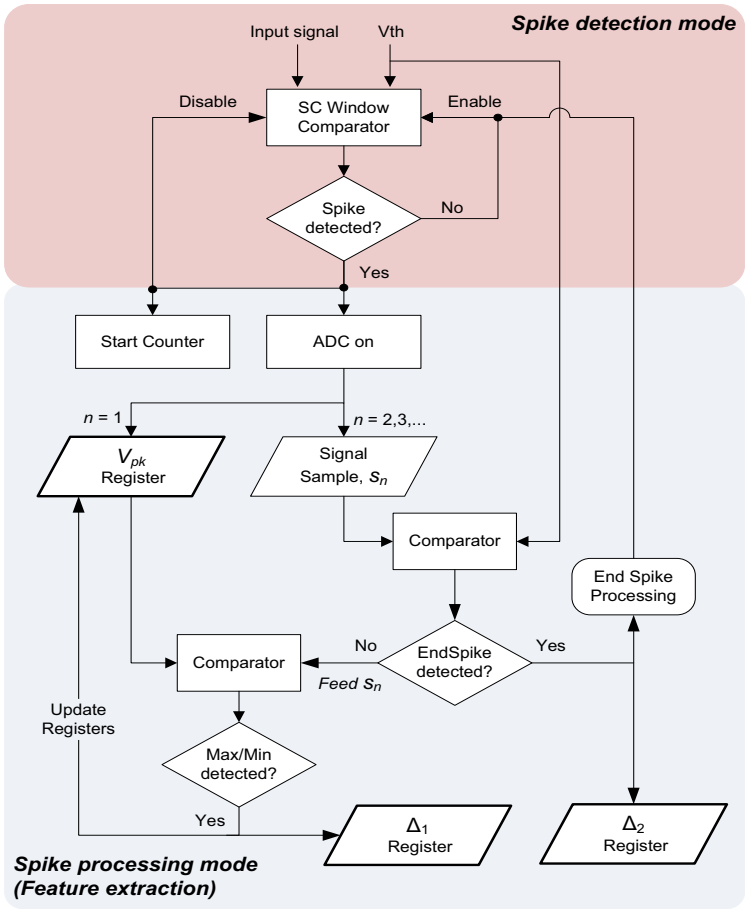

Fig. 2: Functional flow in the feature extraction mode. 


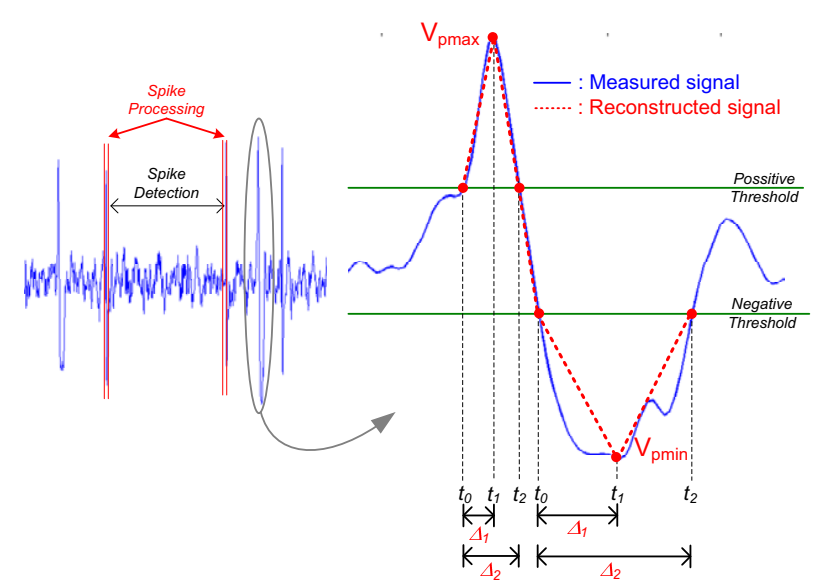

Fig. 3: Feature extraction operation mode.

the spike characterization. The complete ADC (PGA + search algorithm block), working at a clock frequency of $800 \mathrm{kHz}$ (90kS/s throughput rate), is used to keep track of the signal amplitude along the spike. Finally, some registers and digital comparators are used to determine the peak position and the end of the spike phase. The algorithm is illustrated in Fig.2 (only in the case of monophasic spikes, for simplicity).

It is worth observing that the ADC and the window comparator do not operate simultaneously during spike processing, what favors a more homogenous power consumption of the recording channel. Indeed, transistor-level simulations of the whole system, designed in a 130nm CMOS technology at $1.2 \mathrm{~V}$ supply voltage. show that the power consumption remains below $2.1 \mu \mathrm{W}$ during signal tracking and spike detection and only rises up to $3.3 \mu \mathrm{W}$ during spike processing. These results, verified under PVT variations, also include the power contributions of the LNA $(1.6 \mu \mathrm{W})$ and the biasing circuitry.

\section{III.ANALOG-TO-DIGITAL CONVERTER}

Given the requirements on reconfigurability and flexibility to operate at different rates, the ADC is a key element in the channel architecture of Fig. 1. Instead of using a capacitive DAC based SAR ADC as in [1-3], our proposal employs the SC structure of Fig. 4. It consists of a S\&H amplifier/ integrator, a comparator and a passive $\mathrm{SC}$ arrangement.

Circuit operation is based on a conventional binary search algorithm. The signal is first sampled and stored in the integrator by transferring charge from capacitor $C_{i n}$ to $C_{i n t}$. Capacitor $C_{\text {in }}$ is a 3-bit programmable array which allows to scale the input signal, thus providing the ADC with PGA capabilities. Conversion is simply realized by successively comparing the integrated voltage with $V_{r e f} / 2^{n}$, where $V_{r e f}$ is the full-scale reference voltage of the converter, and index $n=$ $1, \ldots, N$, indicates the conversion step $(N$ represents the converter resolution). If the stored voltage is larger (alt., smaller) than $V_{\text {ref }} / 2^{n}$ the comparator sets to '1' (alt., ' 0 ') the $n$-th bit of the conversion, and the integrator is updated by substracting (adding) such increment. As illustrated in the timing diagram of Fig. 5, conversion takes $N+1$ clock cycles,

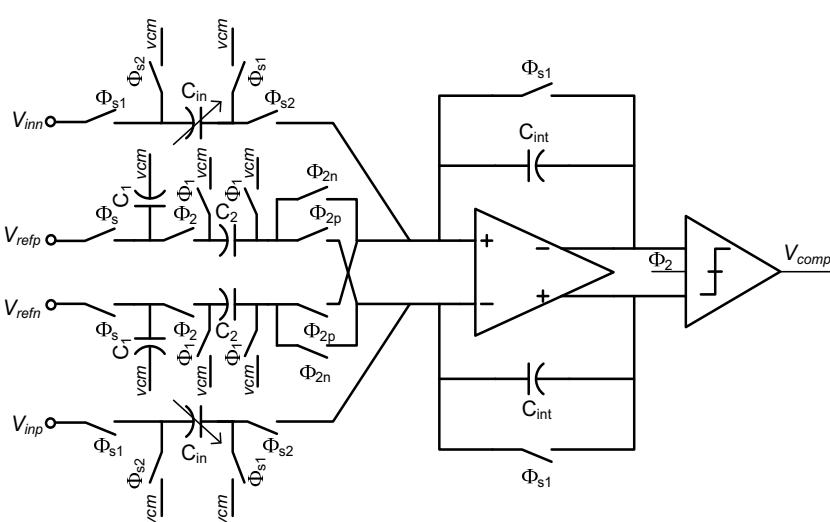

Fig. 4: SC-based ADC schematic.

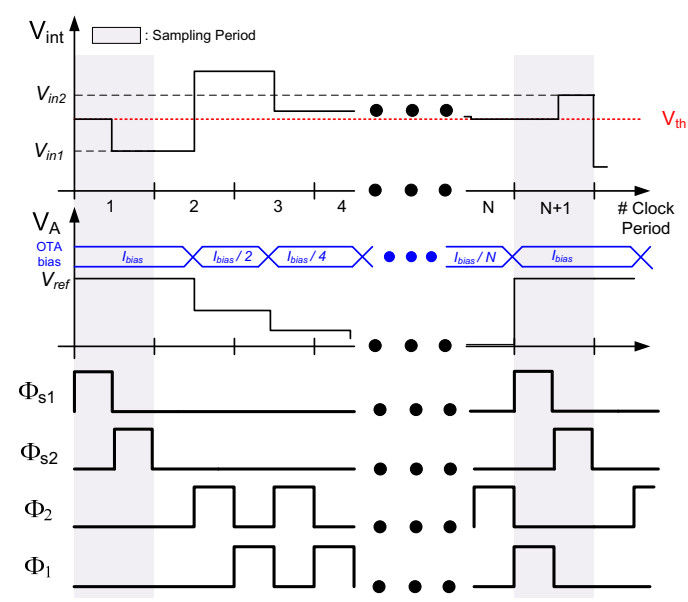

Fig. 5: Clock phases of the SC-based ADC.

the first cycle used for signal sampling.

In the schematic of Fig. 4, the division-by-two operation is simply realized by equally splitting the charge in $C_{1}$ with a nominally identical capacitor $C_{2}$. The addition/substraction operations are simply implemented by means of a switch arrangement which takes advantage of the fully-differential feature of the circuit.

A dynamically-biased folded-cascoded topology has been used to implement the OTA of Fig. 4. As the integrated reference voltage decreases along the conversion process, the OTA biasing current is progressively diminished according to the more relaxed requirements on slew rate performance as the ADC approaches the end of the conversion. In other respects, the comparator is a current-controlled dynamic latch, whose bias current is powered off during the tracking phase for the sake of power saving.

\section{IV.ADC SIMULATION RESULTS}

Fig. 6 shows the layout of the complete ADC. It has been implemented in a $130 \mathrm{~nm}$ CMOS technology and occupies $190 \mu \mathrm{m} \times 225 \mu \mathrm{m}$. It operates from a $1.2 \mathrm{~V}$ supply voltage and features a differential full-scale range of $1.2 V_{\mathrm{pp}}$

Figs. 7 and 8 show two FFT analyses of the ADC at the sampling rates of $90 \mathrm{kS} / \mathrm{s}$ (required during spike processing) 


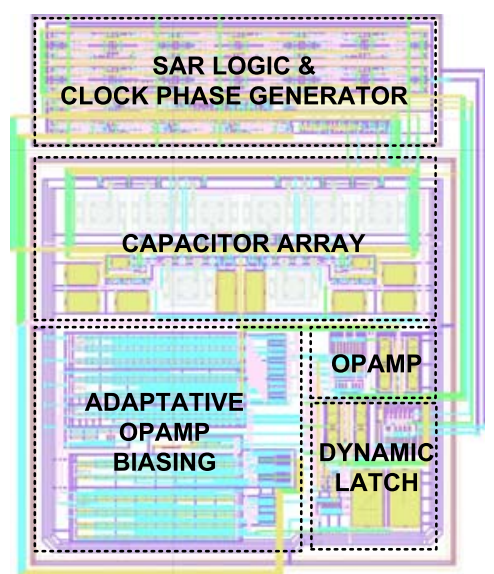

Fig. 6: Layout of the SC-based ADC

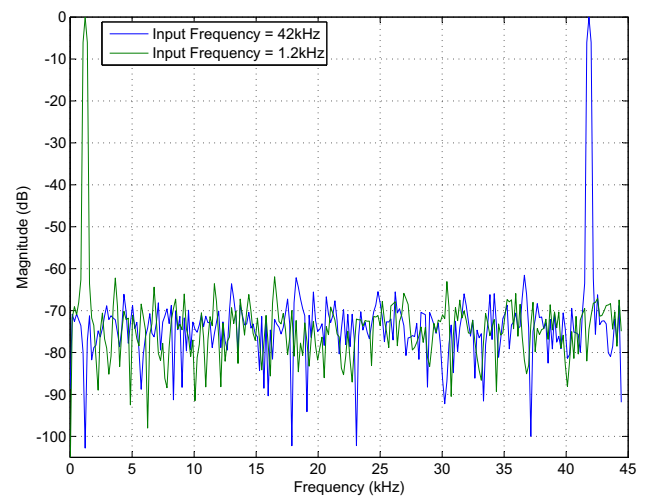

Fig. 7: FFT-spectrum of the ADC output response at $90 \mathrm{kS} / \mathrm{s}$ for low and high sinusoidal input frequencies

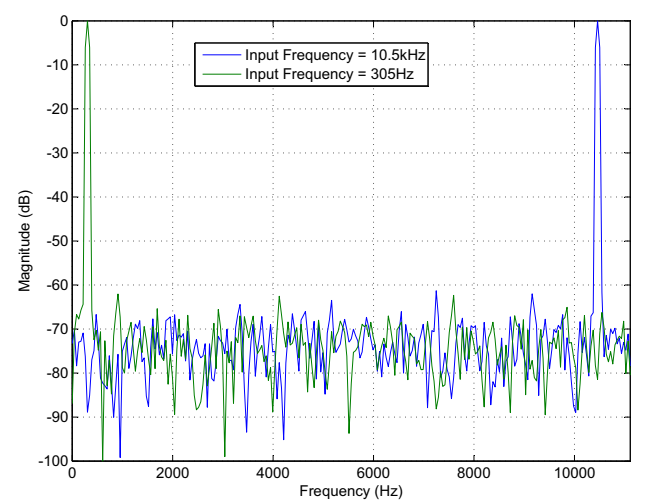

Fig. 8: FFT-spectrum of the ADC output response at $22.5 \mathrm{kS} / \mathrm{s}$ for low and high sinusoidal input frequencies

and $22.5 \mathrm{kS} / \mathrm{s}$ (required during signal tracking), respectively. In both figures, two full-scale input tones are shown; one at low frequencies and the other close to Nyquist rate. Figures show that the $S F D R$ and the $S N D R$ are approximately $62 \mathrm{~dB}$ and $50 \mathrm{~dB}$, respectively, which corresponds to an $E N O B$ of about 8 bits. These features do not vary with the gain of the embedded PGA. At $0 \mathrm{db}$ voltage gain, the equivalent inputreferred noise level of the ADC is $490 \mu V_{\text {rms }}$, well below the quantization step of the converter. The performance of the ADC, obtained at a voltage gain of $18 \mathrm{~dB}$, is summarized in Table I.
TABLE I. Characteristics of the ADC

\begin{tabular}{|l|c|c|}
\hline & $\begin{array}{c}\text { Signal } \\
\text { Tracking }\end{array}$ & $\begin{array}{c}\text { Spike } \\
\text { Processing }\end{array}$ \\
\hline Resolution & \multicolumn{2}{|c|}{8 -bits } \\
\hline Technology & \multicolumn{2}{|c|}{ CMOS $130 \mathrm{~nm}$} \\
\hline Voltage supply & \multicolumn{2}{|c|}{$1.2 \mathrm{~V}$} \\
\hline Conversion Rate & $22.5 \mathrm{kS} / \mathrm{s}$ & $90 \mathrm{kS} / \mathrm{s}$ \\
\hline$E N O B$ & $7.98-\mathrm{bits}$ & $7.96-\mathrm{bits}$ \\
\hline$S N D R$ & $49.8 \mathrm{~dB}$ & $49.68 \mathrm{~dB}$ \\
\hline Power Consumption & $500 \mathrm{nW}$ & $1.8 \mu \mathrm{W}$ \\
\hline
\end{tabular}

\section{V.CONCLUSIONS}

This paper describes the architecture of a neural spike recording channel and presents the design of one of its key elements, a multi-rate, reconfigurable ADC. The channel offers the possibility to record raw data sampled at $22.2 \mathrm{kS} / \mathrm{s}$ with 8-bit precision (signal tracking mode), or perform an inchannel data compression process in which a limited set of PWL parameters is extracted at very spike occurrence (feature extraction mode). The proposed ADC is able to adapt to the different operation frequencies imposed by the channel operation modes and embeds a programmable input stage to scale the voltage gain from 0 to $18 \mathrm{~dB}$ at discrete steps of $3 \mathrm{~dB}$. Moreover, it features a low equivalent input capacitance what relaxes the requirements of the preceding LNA and favors the reduction of area and power consumptions. Active circuits of the ADC are dynamically biased during conversion to reduce the average power consumption of the block. Simulation results from the extracted layout of the ADC give almost 8bits $E N O B$ at $22.5 \mathrm{kS} / \mathrm{s}$ and $90 \mathrm{kS} / \mathrm{s}$ with a power consumption of $500 \mathrm{nW}$ and $1.8 \mu \mathrm{W}$, respectively.

\section{ACKNOWLEDGMENTS}

This work has been supported by the Spanish Ministry of Science \& Innovation under grant TEC2009-08447, the Junta de Andalucía under grant TIC-02818 and the 2007-2013 FEDER Programme.

\section{REFERENCES}

[1] R. R. Harrison, et al., "A Low-Power Integrated Circuit for a Wireless 100-Electrode Neural Recording System," IEEE J. Solid-State Circ., vol. 42(1), pp. 123-133, Jan. 2007.

[2] A. M. Sodagar, et al., "An Implantable 64-Channel Wireless Microsystem for Single-Unit Neural Recording," IEEE $J$. Solid-State Circ., vol. 44(9), pp. 2591-2604, Sept. 2009.

[3] M.S. Chae, et al., "A 128-Channel 6mW Wireless Neural Recording IC With Spike Feature Extraction and UWB Transmitter," IEEE Trans. on Neural Systems and Rehabilitation Eng., vol. 17(4), pp. 312-321, Aug. 2009.

[4] B. Gosselin, et al., "A Mixed-Signal Multichip Neural Recording Interface with Bandwidth Reduction," IEEE Trans. Biomedical Circ. Sys., vol. 3(3), pp. 129-141. June 2009.

[5] S. Gibson, J. W. Judy, et al., "Comparison of spike-sorting algorithms for future hardware implementation," IEEE EMBS Conf., pp. 5015-5020, Aug. 2008.

[6] R. Quian Quiroga, et al., "Unsupervised spike detection and sorting with wavelets and superparamagnetic clustering," Neural Comp. 16, pp. 1661-1687, 2004. 